\title{
The occurrence and quality of care of non-communicable diseases in people living with HIV in Maseru, Lesotho: a mixed-methods study
}

\author{
Eltony Mugomeri ${ }^{1}$, Palesa Khama ${ }^{1, \#}$, Polo C. Seshea ${ }^{1, \#}$, Bisrat Bekele $^{1, *}$, Selatela Mojai ${ }^{1, \#}$, Charles Maibvise ${ }^{2, *}$, \\ Champion N. Nyoni ${ }^{3, *}$
}

${ }^{1}$ Department of Pharmacy, National University of Lesotho, Roma, Lesotho

${ }^{2}$ University of Swaziland, Kwaluseni, Swaziland

${ }^{3}$ Paray School of Nursing, Lesotho

\#These authors contributed equally to this manuscript.

\begin{abstract}
Introduction: Chronic non-communicable diseases (NCDs) constitute a health problem worldwide. This problem is particularly important in people living with human immunodeficiency virus (PLHIV) in sub-Saharan countries where the prevalence of NCDs is expected to increase due to the epidemiological transition of life spans for PLHIV as treatment outcomes for HIV continue to improve. This study is the first to assess the occurrence and quality of care (QoC) of NCDs in PLHIV and to identify the contextual issues underpinning the QoC in Lesotho - a sub-Saharan country with the second highest prevalence of HIV globally.

Material and methods: Based on the Donabedian (2005) model for assessing QoC, the study used a triangulation of quantitative patient responses on QoC of NCDs, their treatment outcomes and qualitative interviews with hospital staff in Maseru, Lesotho.

Results: Two hundred and forty-six PLHIV with a median survival time of 7 years since the first diagnosis of HIV (IQR: 3-9 years) and 25 hospital staff were included in the study. Forty-two percent (42\%) of the patients had at least one diagnosed NCD, and 33\% of these were diagnosed outside of the antiretroviral treatment (ART) centers. Overall, the QoC of NCDs was inadequate. Only 32\% of patients with NCDs had records on NCD treatment outcomes in the ART centers. Shortage and disrepair of equipment, along with inadequate staff were the major barriers affecting the organizational structure for the care of NCDs. Inadequate screening for NCDs, disintegrated checkup schedules for NCDs/HIV co-morbid conditions, and inadequate patient education were the major issues affecting the treatment processes.

Conclusions: The longer life spans of PLHIV in this study emphasises the need to scale up the diagnosis of NCDs while improving their QoC in PLHIV in Lesotho. The issues underpinning the QoC of NCDs should thus be prioritised in the interventions aimed at improving QoC of NCDs in PLHIV.
\end{abstract}

Key words: Donabedian model, HIV, Lesotho, non-communicable diseases, quality of care.

Address for correspondence: Eltony Mugomeri, Department of Pharmacy, National University of Lesotho, Roma Campus, 180 Maseru, Lesotho, e-mail: emugomeri@yahoo.com
Article history:

Received: 10.10.2016

Received in revised form: 29.01.2017

Accepted: 11.02.2017

Available online: 25.05.2017
International Journal of HIV-Related Problems

HIV \& AIDS

R e vi e w 


\section{Introduction}

Chronic non-communicable diseases (NCDs), particularly cardiovascular disease, cancer, chronic respiratory disease, and diabetes are the leading cause of death globally, killing more people each year than all other causes combined [1]. Out of 57 million deaths that occurred globally in 2008, 36 million (63\%) were due to NCDs [2]. The World Health Organization (WHO) estimates that NCDs account for $28 \%$ of the disease burden in Southern Africa [3]. The prevalence of NCDs in people living with human immunodeficiency virus (HIV) in Southern Africa is increasing mainly due to the epidemiological transition, a phenomenon which refers to the general increase in certain NCDs due to longer life spans that are observed in populations with improved treatment outcomes of chronic diseases such as HIV [4]. Therefore, improving quality of care (QoC) of NCDs among HIV positive patients is becoming ever more important.

Due to the high prevalence of HIV, health systems in sub-Saharan Africa are allocating a higher priority to the provision of antiretroviral treatment (ART) services compared to other health care services, including the care of NCDs [5]. It is also not surprising that the QoC for NCDs in most sub-Saharan countries remains understudied [6-8].

According to the literature available, better assessments of QoC of NCDs in people living with HIV (PLHIV) are those that use broad and multi-level approaches [9]. This includes assessing the organizational structure, daily processes and treatment outcomes in health institutions treating NCDs and HIV. Donabedian [9] notes that the assessment of organizational structure should also target the components of the healthcare system, including personnel training, skills, and adequacy of equipment. Figure 1 outlines a conceptual framework for a multi-level assessment approach modified from the Donabedian model [9]. This framework is applicable in HIV endemic areas undergoing epidemiological transitions of life spans for PLHIV. It is important to note that this framework includes the assessment of organization processes, the use of appropriate diagnostic and therapeutic modalities for individual patients, together with the assessment of process outcomes, particularly the consequences of treatment. Yet, studies that utilize this comprehensive approach are scarce in sub-Saharan Africa.

Improving patients' health literacy is also essential for QoC of NCDs in sub-Saharan Africa. Despite the escalating prevalence of NCDs in this region, health literacy, defined by Nutbeam [10] as the level of functional knowledge that patients have for making health-related decisions, remains low [11]. Literacy on drug interactions, adverse reactions, dosages, and the life-long nature of NCDs in PLHIV treatment are key elements of patient education. Kruk et al. [11] emphasize that disaggregated treatment and care of NCDs/HIV in

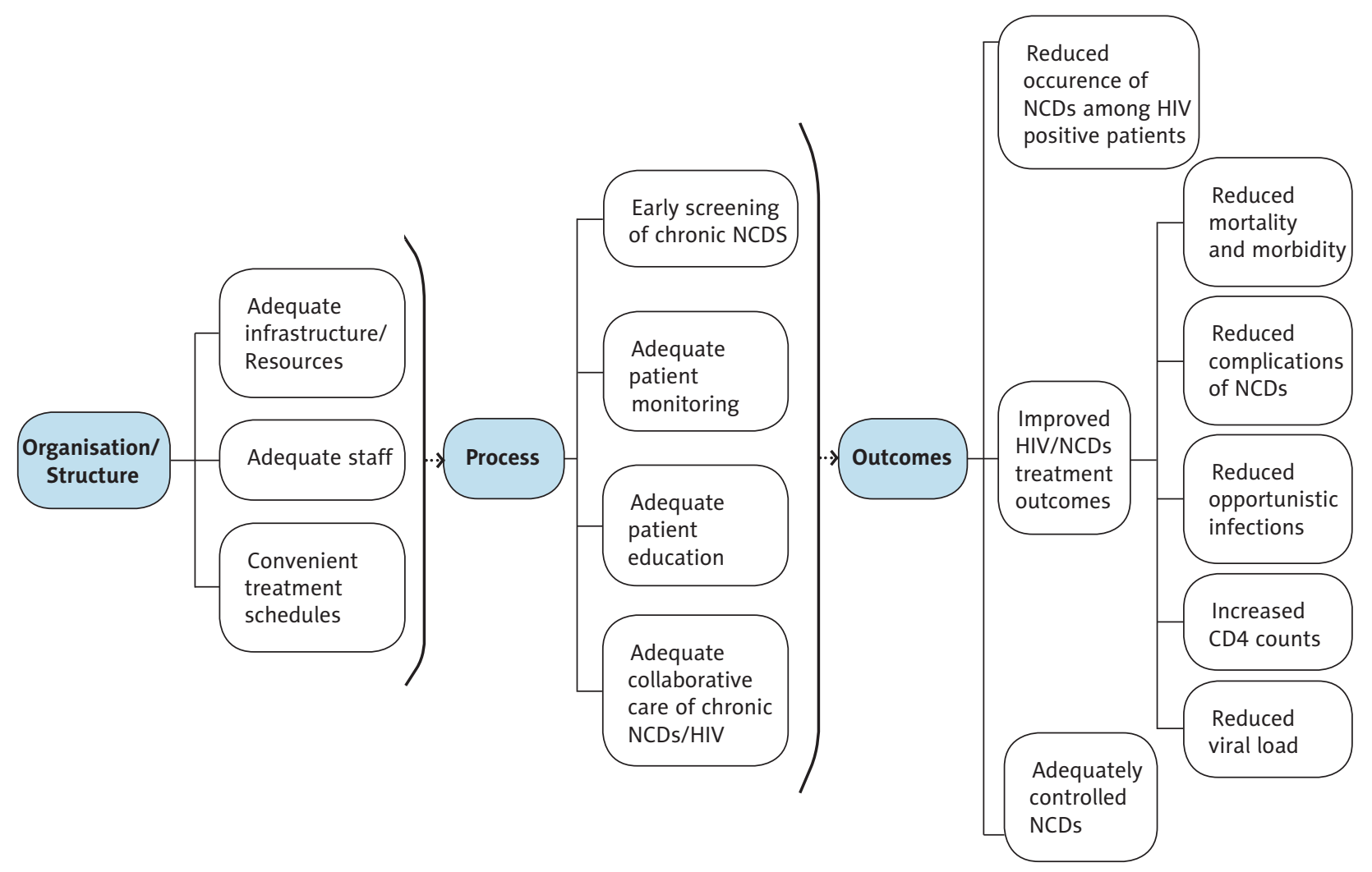

Figure 1. A proposed conceptual framework for assessing the quality of care of chronic non-communicable diseases (NCDs) in countries experiencing epidemiological transition of life spans 
sub-Saharan Africa affects the quality of patient education. Almeida et al. [12] also note that less than $25 \%$ of HIV positive patients have adequate literacy about the consequences of missing ART dosages. Therefore, quality patient education should be prioritized.

Lesotho is classified as one of the least developed countries in sub-Saharan Africa and with an estimated adult HIV prevalence rate of about $23 \%$, has the second highest HIV burden in the world [13]. The literature on the prevalence of individual NCDs in the general population in Lesotho indicates that there is also a high burden of NCDs. The Government of Lesotho (GoL) estimates the prevalence of hypertension (HTN) at about $35 \%$ in adults aged between 40-49 years [14], while that of diabetes is estimated at $2.5 \%$ [15]. Data in other age groups is not available in the country. Heart failure and stroke alone accounted for $8 \%$ of national mortality in 2010 [15]. About $36 \%$ of the population is classified as obese, while $39 \%$ is reported to abuse alcohol The GoL [15] also decries the high rates of smoking, inappropriate diet, lack of physical activity, and road traffic accidents. Hypertension, diabetes mellitus (DM), and cancers are the major NCDs in Lesotho. However, statistics on the prevalence of these NCDs in the HIV positive sub-group is scarce.

The QoC of HIV-positive people who also have NCDs is scarcely documented in Lesotho despite the fact that this research area is listed in the 2013-2018 national research agenda [16]. The literature supports that care for patients with co-morbidities should be integrated to improve the quality of care. Cohen et al. [17] note that poor integrated care of NCDs and HIV, and high nurse workload are major obstacle to integrated NCD/HIV care in Lesotho. This affects the quality of decisions made on diagnosis and monitoring of NCDs in PLHIV.

The purpose of this study was to assess the QoC of NCDs in PLHIV in Lesotho, and to identify contextual issues underpinning the QoC of NCDs in PLHIV in Lesotho.

\section{Material and methods}

\section{Study setting}

The Kingdom of Lesotho, located $29.6100^{\circ} \mathrm{S}, 28.2336^{\circ} \mathrm{E}$ and enclaved by the Republic of South Africa, is divided into 10 administrative districts with the capital city Maseru, being in Maseru District. Maseru district has about 25\% of the country's estimated two million people. The study was conducted at two referral hospitals that provide primary health and HIV care in Maseru District. The district has three district hospitals and five health centers. The two selected referral hospitals were the largest in the district. At both hospitals, HIV care was provided in dedicated ART centers that offer HIV care services only. The total number of active patients in HIV care during the study period according to the ART registers at the two hospitals was 2,700. The prevalence of HIV in Maseru District is about $27 \%$ and is the highest in the country [18].

\section{Study design}

This sequential exploratory study used a triangulation of quantitative and qualitative methods in two phases to obtain a more complete understanding of the research problem [19]. The design of the assessment of QoC of NCDs in the two study phases was informed by the Donabedian assessment of QoC model [9].

Phase one of the study was a cross-sectional survey of the quantitative patient responses on QoC of NCDs based on structured questionnaires, and a review of their treatment outcomes from their medical records for ART. The questionnaire and data extraction form for this phase were developed by the researchers with inputs from hospital staff that had at least five-years working experience at the two hospitals included in the study.

The main sections in the patient questionnaire included demographic characteristics, history of NCDs screening, and health literacy about the NCDs. The patient questionnaire had six structured questions and three open questions. On average, the questionnaire needed 15 minutes to complete. On screening NCDs, patient were asked if they had ever been diagnosed for NCDs and those diagnosed of at least one NCD were asked to respond to follow-up questions including places of diagnosis, checkup, and treatment. Concerning health literacy, three open questions assessed the patients' understanding of their NCDs, the causes, and knowledge of taking their medications. Data on treatment outcomes of NCDs, if any, and ART treatment outcomes were extracted using a data collection form designed by the researchers.

Phase two consisted of cross-sectional interviews with hospital staff on QoC of NCDs based on a semi-structured interview guide. The interview with hospital staff focused on understanding contextual issues underpinning the QoC of NCDs, including organizational structure, relevant processes, patient education, and collaborative care.

\section{The reliability of the patient questionnaire, the interview guide and the medical records}

The patient questionnaire was translated from English to vernacular language (Sesotho) by two language specialists. The questionnaire was pilot tested with five patients at one of the data collection sites; the pilot results were not included in the final analysis. The internal consistency for each section of the data collection tool was tested using the Cronbach's a coefficient [20]. The a coefficient was 0.770 on average per question, implying that the tool was satisfactorily reliable. The interview guide was pilot tested with five staff members at each hospital and their pilot data was also not included in the final data collection.

The study relied on ART records for patients' treatment outcomes. A Microsoft Access database tool was developed to improve data entry and coding of the data. To improve the quality of data capture, data collectors were trained for five days on how to capture the data into the database. 


\section{Study sampling and data collection}

In phase 1, HIV-positive patients attending their checkup visits during the data collection period at the data collection sites were randomly selected, based on a sampling frame created from the patients' daily checkup schedules. Patients meeting the selection criteria were invited to participate based on the minimum sample size of 246 , calculated according to the survey guidelines recommended by the WHO. The sample size was calculated assuming a target population of $2,700 \mathrm{pa}-$ tients at the two hospitals, an error of margin of $5 \%$ at $95 \%$ confidence interval and a minimum response of $50 \%$. However, an additional $10 \%$ over and above the calculated minimum sample size was invited to cater for non-responses. Overall, 271 patients were invited. Every second patient on the sampling frame willing to participate was included in the study. Patients incapacitated due to advanced illness and those who could not respond to the questionnaire were excluded from the study. In total, 250 patient agreed to participate in the study.

In phase two, key informant staff were selected based on work experience at the data collection sites. Nurses, pharmacists, and medical officers with at least 2 years of working experience at the study sites and were willing to participate were included in the study until data saturation was reached. Each of the two hospitals had about 20 staff members working in the ART center. In total, 25 hospital staff members were interviewed at the two study sites.

Data on treatment outcomes of NCDs and HIV were collected by the researchers between February and May 2016 from patients' medical records at the study sites. Patients completed questionnaires on their own with the assistance of data collectors. Data from completed questionnaires and from the patients' medical records were also captured using the Microsoft Access database tool.

\section{Data analysis}

The major outcome variables for evaluating quality of care of NCDs were diagnosis of NCDs or lack thereof; place of diagnosis, checkup, and treatment; frequency of checkup; treatment outcomes; patients' health knowledge about NCDs and prescribed drugs.

Patients' responses on health literacy about NCDs and prescribed drugs were assessed against a set of possible correct answers designed by the researchers, and were scaled using a Likert scale, where a higher score indicated better literacy. The Likert scale ranged from grossly inadequate (1), inadequate (2), average (3), above average (4), and excellent (5). 'Grossly inadequate' was defined as having no information about any of the three questions asked, while 'inadequate' responses corresponded to having some idea about their NCDs and medications, however insufficient. 'Average, 'above average', and 'excellent' were defined as having better understanding of their NCDs and medications, where excellent reflected having sufficient practical knowledge. An overall score was also calculated and classified as bivariate scores either as in- adequate or adequate. An inadequate overall health literacy score was defined as having a score below the average score.

An unknown NCD status referred to a situation where a patient had not been tested for NCDs. The duration of NCDs was defined as the period between the time of diagnosis and the day of data collection, whilst the duration since the last checkup referred to the period between the last NCD checkup date and the day of data collection. Uncontrolled blood pressure (BP) was defined as having BP measurements greater than $140 / 90 \mathrm{mmHg}$ on any visit over the previous six months.

Qualitative interview data obtained from the staff on the assessment of the major barriers affecting the organizational structure for the care of NCDs were analyzed through axial coding. Core themes from the interviews were sorted into two categories, namely organizational structure issues and relevant processes affecting the QoC of NCDs.

Data from the hospital staff interviews were translated to English. Quantitative data for the patients were verified, cleaned, and analyzed. Patient characteristics, including demographic, clinical variables, duration of NCDs, and duration since last checkup were summarized, with frequencies and percentages using Stata version 13 software. The significance of the relationship between HIV and NCDs treatment outcomes were tested using $\chi^{2}$ test at $95 \%$ significance level.

\section{Ethical considerations and clearance}

The study was approved by the Ethics Committee of the Ministry of Health of Lesotho. The participants were provided with information on the background of the study. They were informed that their participation was voluntary and that they could decline to answer certain questions, including the right to withdraw their participation at any time. In addition the information collected was kept confidential. Each participant signed a written consent form before data collection.

\section{Results}

\section{Demographic characteristics of the participants}

A total of 246 PLHIV with a median survival time of 7 years since the first diagnosis of HIV (IQR: 3-9 years) had data acceptable for analysis. The ages of the patients ranged from 22 to 77 years, with a median age of 47 . Seventy $(28.5 \%)$ participants were aged less than 40, 132 (53.6\%) were between 40 and 60 , and $44(17.9 \%)$ were above 60 in age.

One hundred and sixty (76.2\%) participants were female, while $50(23.8 \%)$ were male. Thirty-six did not indicate their sex. One hundred and fifteen (46.7\%) participants were married, $82(33.3 \%)$ were widowed, while $31(12.7 \%)$ had never married. Only 18 (7.3\%) were divorced. The most common (89.1\%) entry point into the ART center was the outpatient department (OPD). Only 23 (10.9\%) had transferred from other health institutions. 


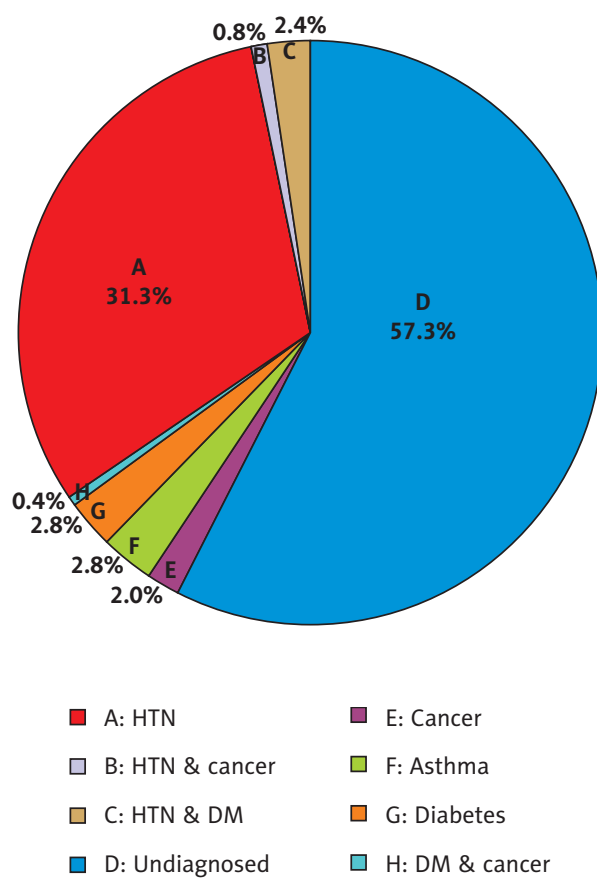

Figure 2. Distribution of non-communicable diseases (NCDs) $(n=246)$

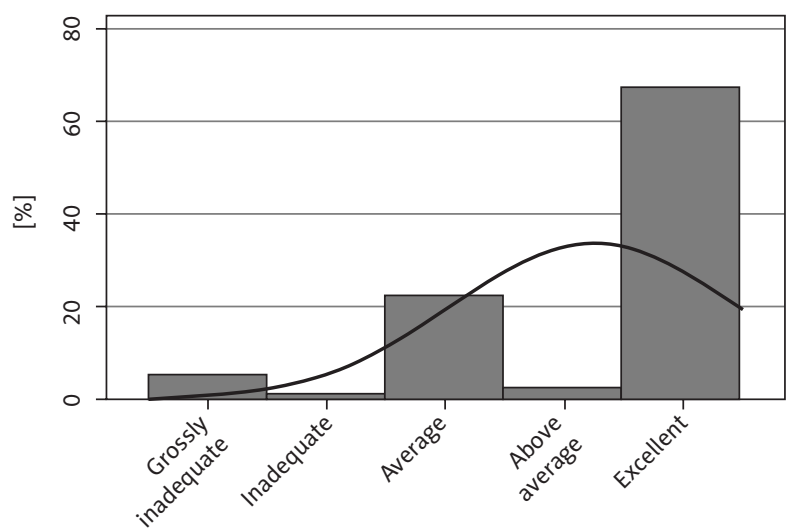

Score (Definition of NCD)

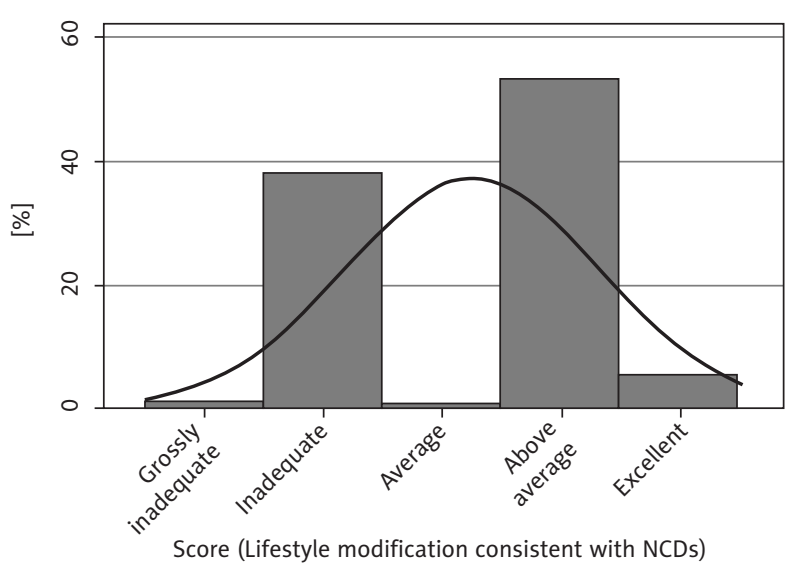

\section{The occurrence of NCDs and places of diagnosis, treatment and checkup}

Overall, 104 (42.3\%) patients were diagnosed with at least one NCD. Of these, $92.3 \%$ had mono-condition, while $7.7 \%$ had multiple NCDs. Figure 2 shows the percentage distribution of the NCDs among the study population. The categories ranged from those with multiple comorbidity of DM/ cancer $(0.4 \%, n=104)$ to HTN $(31.3 \%)$. About $90 \%(n=14)$ of the patients diagnosed with diabetes had type I DM, while $62.5 \%(n=8)$ and $37.5 \%$ of the patients diagnosed with cancer had cervical and breast cancer, respectively.

Seventy (67.3\%) patients were diagnosed with NCDs at the ART center, and 34 (32.7\%) were diagnosed at other health centers. One hundred and two (98.1\%) patients indicated that they were receiving treatment and checkup outside their ART center.

\section{Frequency of checkup for BP and blood sugar}

Out of 85 patients who were diagnosed of HTN, only 15 (17.6\%) had been checked for BP in the last 3 months, while $16(9.9 \%)$ of the 161 who were not diagnosed with HTN had been diagnosed in the last three months. Out of 14 patients who were diagnosed of DM, only 5 (35.7\%) had been

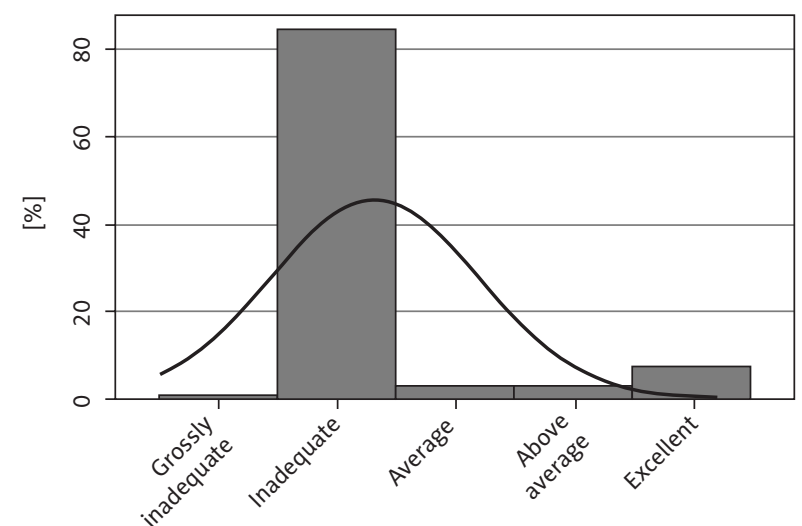

Score (Causes of NCD)

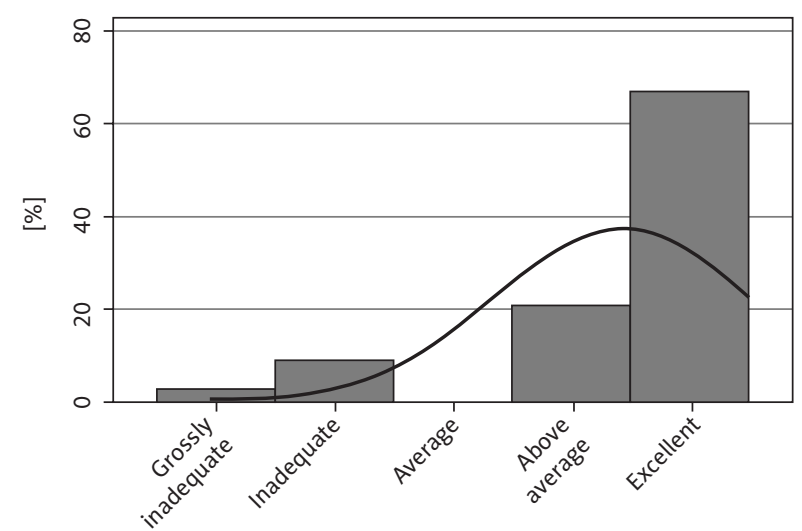

Score (Knowledge of complications of NCDs)

Figure 3. Distribution of the patients' responses on health literacy 
checked for blood glucose in the last 3 months, while only $26(13.8 \%)$ of the 189 not diagnosed with DM had their blood glucose measured in the last three months.

\section{Patients' literacy on NCDs}

Out of the 104 patients who had at least one NCD diagnosed, the lowest scores on health literacy were recorded in the category on cause of their NCDs, where $84.6 \%$ scored below the average score, followed by the category on lifestyle modification, where $39.4 \%$ scored below average (Figure 3 ). The highest scores were recorded in the category on complications of their NCDs, where $87.5 \%$ scored above average.

\section{Treatment outcomes}

Only 35 (41.2\%) out of 85 patients who were diagnosed of HTN had results for treatment outcomes available. Of these, $65.7 \%$ had their BP under control (BP $<140 / 90 \mathrm{mmHg}$ ). In total, $25.7 \%$ had BP levels between $140 / 90$ and $160 / 100$ $\mathrm{mmHg}$; 5.7\% ranged between $160 / 100$ and $180 / 120 \mathrm{mmHg}$, while $2.9 \%$ had BP levels over 180/120 mmHg. Overall, $211(85.8 \%)$ who had no blood pressure results had left their records on BP measurements at home.

CD4 count as an indicator of HIV treatment outcomes was not significantly $(p=0.654)$ associated with hypertension treatment outcomes. However, 9 (27.3\%) out of 33 patients with $\mathrm{CD} 4<500$ cells $/ \mathrm{mm}^{3}$ had uncontrolled blood pressure.

\section{Factors affecting QoC of NCDs}

Twenty-five key staff informants comprising 16 (64\%) nurses, 5 (20\%) pharmacists, and 4 (12.7\%) doctors participated in the study at the two study sites. Their ages ranged from 24 to 58 (median, 36; inter-quartile range [IQR] = 22). Their work experience ranged from 2 to 30 years (median, 6; $\mathrm{IQR}=14$ ).

A number of barriers to the diagnosis and management of NCDs in the adopted framework by Donabedian [9] were identified from the responses of the key staff informants at the ART centers. The shortage of staff, inadequate equipment, and disrepair, particularly sphygmomanometers and glucometers were the major barriers affecting organizational structural component of the framework adopted for the study.

Disintegrated checkup schedules for NCDs and HIV was a major issue affecting the 'processes' component of the Donabedian framework [9]. One study site did not screen and treat NCDs. All the participants at this study site emphasized that patients had to get screened and treated elsewhere outside the ART center, unless they were symptomatic of NCDs. In the other study site, participants had to get screened and treated for hypertension or diabetes at the diabetes or hypertension clinic located in the same hospital. However, the checkup schedules for NCDs and HIV for those with NCDs/HIV co-morbid conditions did not correspond.
Lack of collaboration between ART centers and NCD clinics, particularly on clinical records was another major issue affecting the component of processes in the framework by Donabedian [9]. The staff at the two study sites reported that it was not mandatory for patients with NCDs/HIV to bring their NCDs treatment records to the ART centers at all times, unless asked to do so by the clinicians. Participants at the two study sites also reported that patient education was mainly given at the time of diagnosis of NCDs. After the initial diagnosis of NCDs, ART staff rarely offered further patient education on NCDs due to time constraints.

\section{Discussion}

\section{Overview of the results}

The prevalence of NCDs in HIV-positive patients in this study was $42.3 \%$. However, about $58 \%$ had unknown NCD status. This proportion implies that a significant number of patients need to be screened for NCDs during their monthly visits at the ART centers. Mugomeri et al. [8] note that late diagnosis of NCDs increases the occurrence of complications, including organ damage, stroke, heart failure, or renal failure. Therefore, further studies to determine the prevalence of NCDs in people living with HIV are required in this population. Notably, this research gap has been prioritized by the Government of Lesotho in its 2013-2018 national research agenda [16]. However, the $42.3 \%$ prevalence observed in this study is high, notwithstanding the high proportion of patients with unknown NCD status.

The key informant staff included in this study reported that organizational structure challenges, including shortage of equipment and staff, and poorly maintained equipment were the major obstacles hindering the screening of NCDs. Lack of collaborative care between ART and NCDs clinics was another challenge. Janssens et al. [6], Govindasamy et al. [21], and Mateen et al. [22] note that integrated care models significantly improve QoC of NCDs.

About $92 \%$ of the patients diagnosed with NCDs had mono-NCD condition of HTN, while $8 \%$ had multiple NCDs. The overall prevalence of HTN in this study was $31 \%$. This proportion is lower than the $41 \%$ reported by GoL [15] in the general population. Rabkin and El-Sadr [23] note that the prevalence of NCDs in HIV-positive patients may be higher than in the general population due to the epidemiological transition phenomenon. Hypertension in HIV is exacerbated by many factors, including the long-term effects of HIV infection and ART [24]. In particular, dyslipidemia due to ART and the use of protease inhibitors increase the occurrence of $\operatorname{HTN}[25,26]$.

A higher proportion of patients with undiagnosed NCDs, particularly HTN and DM, had not been checked for BP and blood glucose in the last three months. In addition, about $34 \%$ of patients with adequate blood pressure data had uncontrolled blood pressure (> 140/90 mmHg). Only 33 (31.7\%) had adequate data on blood pressure for analysis of HTN 
treatment outcomes. Therefore, there was insufficient data to obtain a decisive outcome on this variable. However, Thinyane et al. [27] note that about $75 \%$ of HTN patients have inadequately controlled blood pressure in the general population in Lesotho. This further emphasizes the need to scale up the screening and monitoring of HTN in people living with HIV.

Diabetes and asthma had a similar prevalence of about $3 \%$. The GoL [15] also estimates the prevalence of diabetes at about $3 \%$ in the general population. The prevalence of DM and asthma in this study excludes the majority (58\%) of patients not screened for NCDs. Therefore, with further screening, there is a probability of obtaining a higher prevalence of DM and asthma in this population. This highlights the need to scale up the screening of these conditions. Brown et al. [28] note that the incidence of diabetes in HIV-infected patients with HAART exposure may be greater than 4-times that of HIV-negative patients.

Only about $67 \%$ of the patients diagnosed with NCDs were diagnosed for NCDs at the two health centers, and $98 \%$ of these patients did not get treatment and monitoring of their NCDs at the ART centers. The lack of screening and monitoring of NCDs at the ART centers, creates many treatment schedules for patients, and may lead to higher defaulting rates in cases where patients have limited funds for transport fares. High transport costs and medical bills for patients with NCDs/HIV co-morbid conditions are an important factor affecting the QoC of NCDs/HIV. Ama de-Graft [29] in Ghana report that failure to keep up with treatment schedules and pay medical bills are a challenge in African settings.

About $85 \%$ of the respondents had inadequate health literacy about the cause of their NCDs, while about $39 \%$ had inadequate health literacy on lifestyle modifications. Causes of NCDs are related to lifestyle. Therefore, the findings in this study where more patients had inadequate knowledge of lifestyle compared to knowledge of causes of NCDs were an anomaly. This anomaly may be an inherent in this study population. However, this highlights that there is need to improve patient education in this population. Mendis et al. [30] emphasizes the need to scale up patient education to improve the patients' levels of health literacy about their NCDs and their respective treatments. Mugomeri et al. [8] and Kilic et al. [31] also corroborate this need in Lesotho.

This study emphasizes that there is a need to improve the QoC of PLHIV and NCDs in Lesotho. The shortage and disrepair of equipment, and inadequate staff are important barriers to QoC of NCDs associated with the organizational structure in this population. In 2006, the number of anti-retroviral therapy (ART) patient consultations per nurse was estimated at 45, with the WHO recommendation of maximum of 30 per day [17]. Similarly, inadequate screening for NCDs, disintegrated checkup schedules for NCDs/HIV co-morbid conditions, inadequate patient education, and poor collaboration between ART centers and NCDs clinics are the major issues affecting the treatment processes. These gaps in the organizational structure and processes may be contributing to poor treatment outcomes and consequently poor QoC of NCDs/HIV. Therefore, interventions targeting the provision of adequately maintained equipment and appropriate staff are required to tackle the problem of organizational structure first and foremost. Adequate correction of problems with organizational structure may help address some of the challenges observed in the treatment processes, particularly the problem of inadequate screening and monitoring of NCDs. These efforts may subsequently result in improved treatment outcomes.

This study also highlights that new models of care for people living with HIV and NCDs are needed in sub-Saharan Africa including Lesotho. Van Olmen et al. [5], Rabkin et al. [32], and Kruk et al. [11] emphasize that health systems in sub-Saharan Africa need to adopt new strategies that leverage the essential elements of chronic HIV care to improve the QoC of chronic NCDs and HIV. These authors also stress the need to improve health literacy of the patients and enable self-management. To achieve this in Lesotho, there is a need to strengthen the primary care services and prioritize investments in laboratory equipment, increasing staff complement, and counselling services. Addressing these issues is required to improve the organizational structure, daily processes, and treatment outcomes in health institutions treating NCDs and HIV [9].

\section{Limitations}

Inadequate screening of NCDs in people living with HIV was a major limitation in this study. This study highlights the urgent need to address this research gap in this population. It is also important to note that social desirability in patients' responses may have influenced their answers. In addition, the results of this study are specific to the Maseru District and may not be generalizable to other districts in the country. The need to carry out similar studies in other districts of the country is therefore emphasized. Furthermore, the outcome variables for measuring QoC of NCDs that were considered in this study were far from being exhaustive. Further studies that consider more variables are therefore required. The main strength of this study is that it provides evidence that informs policy on treatment and monitoring of NCDs/HIV co-morbid conditions.

\section{Conclusions}

The major highpoint of this study is that more needs to be done to improve the diagnosis and QoC of NCDs in Maseru, Lesotho. The fact that a considerable proportion of PLHIV have at least one diagnosed NCD and that a significant share of these patients are diagnosed with NCDs outside of the ART centers emphasises the need to scale up the diagnosis of NCDs in ART centres and improve the QoC of PLHIV and NCDs in this population. The major barriers affecting the organizational structure and treatment processes for the diagnosis and care of NCDs point to the existence of shortcomings in the current model of care for PLHIV and 
NCDs in the country. The need to address these gaps is compelling given the projected increase in life spans for PLHIV as antiretroviral treament outcomes continue to improve.

\section{Recommendations}

This study underscores the need to improve the QoC of HIV-positive patients with NCDs in the country. Particular interventions required include the improvement of staff complements and the provision of adequately maintained equipment. In addition, it is essential to implement the lessons learnt in HIV care to improve the QoC of chronic NCDs. Furthermore, the need to strengthen the primary care services and prioritize investing in laboratory equipment, increasing staff complement, and scaling up counselling services cannot be overemphasized.

\section{Acknowledgements}

We thank the hospital authorities for approving this study and the study participants. We also thank data collectors.

\section{Conflict of interest}

The author's declared no potential conflicts of interest with respect to the research, authorship, and/or publication of this article.

\section{References}

1. Alwan A; World Health Organization. Global status report on noncommunicable diseases 2010. World Health Organization, Geneva 2011.

2. WHO. A global brief on hypertension: silent killer, global public health crisis. World Health Organization, Geneva 2016.

3. Longo D, Fauci A, Kasper D, et al. Harrison's Principles of Internal Medicine. 18th ed. McGraw-Hill Professional, 2011.

4. Schmidt MI, Duncan BB, Azevedo e Silva G, et al. Chronic noncommunicable diseases in Brazil: burden and current challenges. Lancet 2011; 377: 1949-1961.

5. Van Olmen J, Schellevis F, van Damme W, et al. Management of chronic diseases in sub-Saharan Africa: cross-fertilisation between HIV/AIDS and diabetes care. J Trop Med 2012; 2012: 349312.

6. Janssens B, van Damme W, Raleigh B, et al. Offering integrated care for HIV/AIDS, diabetes and hypertension within chronic disease clinics in Cambodia. Bull World Health Organ 2007; 85: 880-885.

7. Mayosi BM, Flisher AJ, Lalloo UG, et al. The burden of non-communicable diseases in South Africa. Lancet 2009; 374: 934-947.

8. Mugomeri E, Ramathebane MV, Maja L, et al. Knowledge of disease condition and medications among hypertension patients in Lesotho. J Am Soc Hypertens 2016; 10: 41-46.

9. Donabedian A. Evaluating the Quality of Medical Care. Milbank Q 2005; 83: 691-729.

10. Nutbeam D. The evolving concept of health literacy. Soc Sci Med 2008; 67: 2072-2078.

11. Kruk ME, Nigenda G, Knaul FM. Redesigning primary care to tackle the global epidemic of noncommunicable disease. Am J Public Health 2015; 105: 431-437.

12. Almeida RF, Vieira AP. Evaluation of HIV/AIDS patients' knowledge on antiretroviral drugs. Braz J Infect Dis 2009; 13: 183-190.
13. WHO. Lesotho factsheets of health statistics. Regional office for Africa: World Health Organisation. 2014. Available at: http:// www.aho.afro.who.int/profiles_information/images/3/3a/LesothoStatistical_Factsheet.pdf (accessed 20.06.2015).

14. Government of Lesotho. Lesotho - Demographic and Health Survey 2009. Ministry of Health and Social Welfare, Maseru 2009/2010.

15. Government of Lesotho. Lesotho - Demographic and Health Survey 2011. Ministry of Health and Social Welfare, Maseru 2011.

16. Government of Lesotho. Health research agenda for Lesotho (2013-2018). Available at: http://www.health.gov.ls/publications/Research/NHRP/RESEARCH\%20AGENDA\%202013-18.pdf (accessed 14.06.2016).

17. Cohen R, Lynch S, Bygrave H, et al. Antiretroviral treatment outcomes from a nurse-driven, community-supported HIV/AIDS treatment programme in rural Lesotho: observational cohort assessment at two years. J Int AIDS Soc 2009; 12: 23.

18. Government of Lesotho. Lesotho global aids response report 2015. Available at: http://www.unaids.org/sites/default/files/media_asset/JC2702_GARPR2015guidelines_en.pdf (accessed 14.06.2016).

19. Creswell JW. Research design: Qualitative, quantitative, and mixed methods approaches. SAGE Publications, Inc., 2013.

20. Tavakol M, Dennick R. Making sense of Cronbach's alpha. Int J Med Educ 2011; 2: 53-55.

21. Govindasamy D, Kranzer K, van Schaik N, et al. Linkage to HIV, TB and non-communicable disease care from a mobile testing unit in Cape Town, South Africa. PLoS One 2013; 8: e80017.

22. Mateen FJ, Kanters S, Kalyesubula R, et al. Hypertension prevalence and Framingham risk score stratification in a large HIV-positive cohort in Uganda. J Hypertens 2013; 31: 1372-1378.

23. Rabkin M, El-Sadr WM. Why reinvent the wheel? Leveraging the lessons of HIV scale-up to confront non-communicable diseases. Global Public Health 2011; 6: 247-256.

24. Crane HM, Van Rompaey SE, Kitahata MM. Antiretroviral medications associated with elevated blood pressure among patients receiving highly active antiretroviral therapy. AIDS 2006; 20: 1019-1026.

25. Fourie CMT, Van Rooyen JM, Kruger A, et al. Lipid abnormalities in a never-treated HIV-1 subtype C-infected African population. Lipids 2010; 45: 73-80.

26. Garg H, Joshi A, Mukherjee D. Cardiovascular complications of HIV infection and treatment. Cardiovasc Hematol Agents Med Chem 2013; 11: 58-66.

27. Thinyane KH, Mothebe T, Sooro M, et al. An observational study of hypertension treatment and patient outcomes in a primary care setting. Pan Afr Med J 2015; 20: 424.

28. Brown TT, Cole SR, Li X, et al. Antiretroviral therapy and the prevalence and incidence of diabetes mellitus in the multicenter AIDS cohort study. Arch Intern Med 2005; 165: 1179-1184.

29. De-Graft Aikins A, Kushitor M, Koram K, et al. Chronic non-communicable diseases and the challenge of universal health coverage: insights from community-based cardiovascular disease research in urban poor communities in Accra, Ghana. BMC Public Health 2014; 14 (Suppl 2): S3.

30. Mendis S, Abegunde D, Oladapo O, et al. Barriers to management of cardiovascular risk in a low-resource setting using hypertension as an entry point. J Hypertens 2004; 22: 59-64.

31. Kilic M, Uzunçakmak T, Ede H. The effect of knowledge about hypertension on the control of high blood pressure. International Journal of the Cardiovascular Academy 2016; 2: 27-32.

32. Rabkin M, Melaku Z, Bruce K, et al. Strengthening health systems for chronic care: leveraging HIV programs to support diabetes services in Ethiopia and Swaziland. J Trop Med 2012; 2012: 137460. 